See discussions, stats, and author profiles for this publication at: https://www.researchgate.net/publication/337221644

\title{
Features and psychometric properties of the Montreal Cognitive Assessment: Review and proposal of a process-based approach version (MoCA-PA)
}

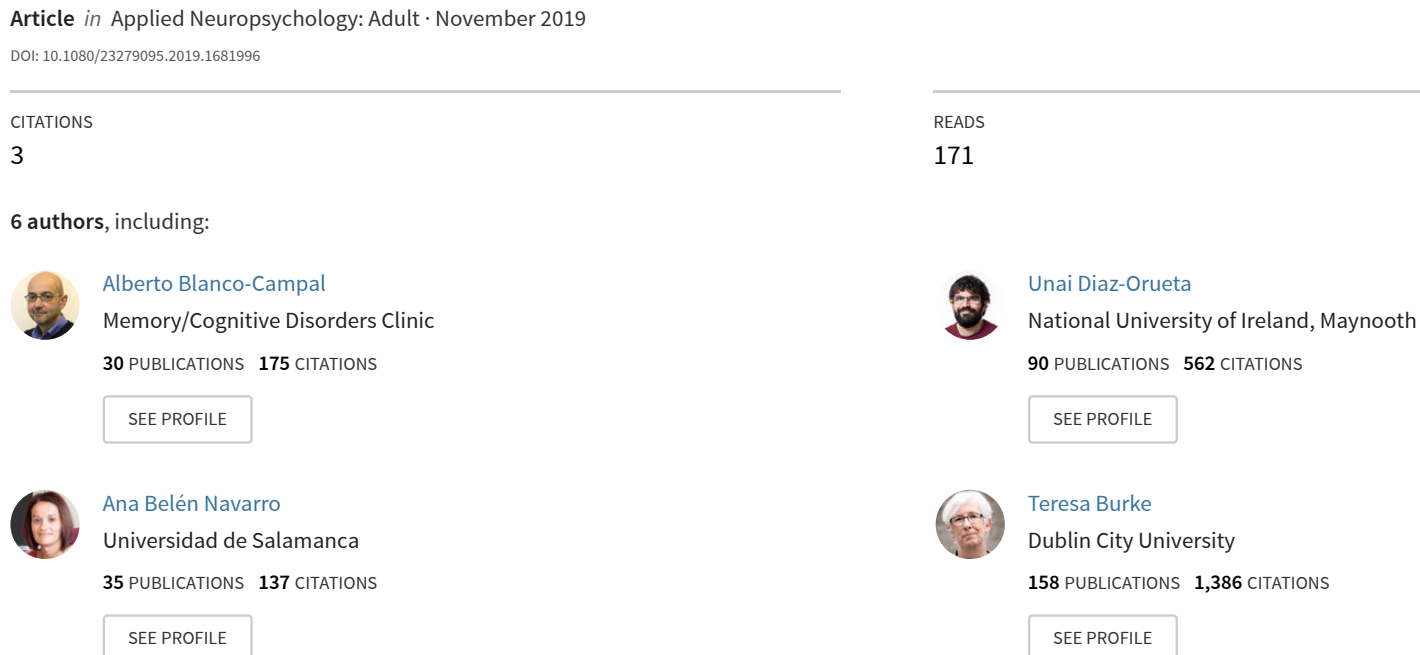

Some of the authors of this publication are also working on these related projects:

i2home View project

E-SPACE: European Standardised Process-Approach to Cognitive Evaluation in Older People View project 


\title{
Features and psychometric properties of the Montreal Cognitive Assessment: Review and proposal of a process-based approach version (MoCA-PA)
}

\author{
Alberto Blanco-Campal ${ }^{a}$, Unai Diaz-Orueta ${ }^{b}$ (D) Ana Belen Navarro-Prados ${ }^{c}$ (D), Teresa Burke ${ }^{d}$, David J Libon ${ }^{e}$, \\ and Melissa Lamar ${ }^{\mathrm{f}}$ \\ ${ }^{a}$ Department of Psychiatry for the Older Person, Co. Louth (Ardee) and Co. Meath (Navan) Memory Clinic Services, Health Service Executive \\ (HSE), Ireland; 'bepartment of Psychology, Maynooth University, Maynooth, Co. Kildare, Ireland; 'Department of Developmental and

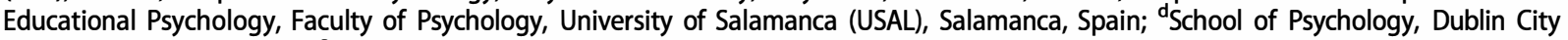 \\ University, Dublin, Ireland; ${ }^{\mathrm{N}}$ New Jersey Institute for Successful Aging, School of Osteopathic Medicine - Rowan University, Stratford, New

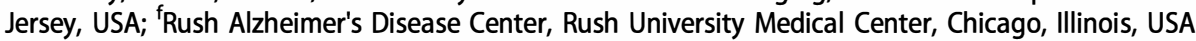

\begin{abstract}
The current study presents a rapid review of the psychometric features of the standard Montreal Cognitive Assessment (MoCA), and the proposal for a modified version of the test, informed by the methodology of the Boston Process Approach to neuropsychological assessment. In order to aid the process of identification of the primary underlying neurocognitive mechanism responsible for defective test performance, the MoCA-Process-Based Approach (MoCA-PA) adds complementary or satellite test conditions in some of its subtests, includes "new" qualitative indices to capture the cognitive processes involved in each cognitive task, and incorporates new qualitative classifications of error subtypes. It provides concurrent assessment of multiple cognitive processes within each task, without significantly increasing administration time or placing significant additional burden upon the respondent. We present preliminary results obtained from an initial sample of 45 community-dwelling older adults attending a University program for seniors. Results suggest the usefulness of additional indices in providing additional information on cognitive deterioration that may be overlooked with the only consideration of quantitative scores. Future research will aim to collect normative data for different clinical populations using the newly developed indices in order to determine the validity and clinical utility of the relatively novel qualitative process-based methods used in the MoCA-PA.
\end{abstract}

\section{KEYWORDS}

Boston Process Approach; cognitive screening; dementia; mild cognitive impairment; Montreal Cognitive Assessment; process-based approach; qualitative analysis of test performance

\section{Introduction}

The Montreal Cognitive Assessment (MoCA) (Nasreddine et al., 2005) is a brief cognitive screening instrument, developed to assist first-line physicians in detecting mild cognitive impairment (MCI), an intermediate clinical state between normal cognitive aging and mild dementia (Geslani, Tierney, Herrman, \& Szalai, 2005; Petersen et al., 2001). Partly due to its clinical value over the Mini-Mental State Examination (MMSE; Folstein, Folstein, \& McHugh, 1975 ) in detecting MCI in a variety of settings and clinical populations (Smith, Gildeh, \& Holmes, 2007; Zadikoff et al., 2008), the MoCA has gained substantial popularity and has been translated into numerous languages.

In line with the vast majority of existing screening tools employed in the detection of MCI and dementia (Appels \& Scherder, 2010; Cullen, O'Neill, Evans, Coen, \& Lawlor, 2007; Milne, Culverwell, Guss, Tuppen, \& Whelton, 2008), the MoCA follows a data driven or quantitative-only method of scoring and interpretation whereby each individual task yields a single achievement score based on the number of correct responses and an aggregate or total test score is derived by simply adding these individual scores, and interpreted in relation to a pass/fail cut-point derived from appropriate normative data. Using this method of interpretation, cognitive impairment is inferred when the achieved score deviates from that of the reference norms. To this end, two recent population-based normative studies held respectively in Ireland and the United States (Kenny et al., 2013; Rossetti, Lacritz, Cullum, \& Weiner, 2011) provide age and education corrected norms, which can assist in the process of detection of cognitive impairment that moves beyond the age-based norms currently available.

While a psychometrically determined test score can empirically demonstrate the relative success of test performance, indicating whether the score falls within the mildly, moderately or severely impaired range in reference to adequate norms, it affords a minimal amount of information regarding the cognitive process recruited to achieve the score or the relationship between the underlying brain dysfunction responsible for the primary cognitive deficit that is contributing to the defective performance on a given

CONTACT Unai Diaz-Orueta unai.diazorueta@mu.ie Department of Psychology, John Hume Building, North Campus, Maynooth University, Maynooth, Co. Kildare, Ireland.

$(6$ Supplemental data for this article can be accessed here.

(c) 2019 Taylor \& Francis Group, LLC 
cognitive task (Kaplan, 1988). To illustrate, two patients with different underlying neuropathology may obtain exactly the same score in a cognitive test but the way in which they approach the task, the types of errors made, as well as the specific underlying cognitive strategies that they recruit to attain the score, may be very different. By going beyond a single summary score, examiners may attempt to interpret the MoCA by exploring the individual scores on each cognitive subdomain in order to draw inferences regarding the respondent's cognitive profile, outlining the cognitive strengths and limitations. However, this practice may lead to erroneous clinical conclusions if one ignores two fundamental issues.

First, although each one of the MoCA cognitive tasks is subsumed under a single cognitive subdomain in the test record form, giving the impression of measuring a single cognitive function, these tasks are in fact multifactorial, requiring a host of cognitive skills for their successful completion. As described in the instructions, time to administer is approximately 10 minutes and the total possible score is 30 points. It purports to assess six different cognitive domains: (1) short-term memory; (2) visuospatial abilities; (3) executive functioning; (4) attention, concentration, and working memory; (5) language; and (6) orientation to time and place. Paradoxically, however, these domains do not map directly onto the tests' subheadings on the MoCA test record form in which visuospatial/executive is a combined heading, and naming, memory, attention, language, abstraction, delayed recall, and orientation are the other headings (Coen, Robertson, Kenny, \& King-Kallimanis, 2016). Hence, respondents may fail the task for a variety of reasons and, without further data, it is difficult to attribute failure to deficits in any particular cognitive process. For example, while the Clock Drawing Test (CDT) is subsumed under visuoconstructional skills, this test is known to require multiple cognitive skills including, among others, language, attention, executive planning, visuoperception and organization, and semantic memory (Ahmed et al., 2016; Cosentino, Jefferson, Chute, Kaplan, \& Libon, 2004; Libon, Malamut, Swenson, Sands, \& Cloud, 1996). Second, like so many other screening tools, since the "standard MoCA" employs a single achievement score for each task, it fails to capture the collection of cognitive functions necessary for accurate completion as well as to provide useful data to identify the primary neurocognitive mechanisms responsible for defective performance.

Despite these observations, it should be noted that the MoCA does not, nor was it created to be, a measure of individual cognitive domains; however, given its widespread use, considering ways to extrapolate more information on such distinctions would be a significant contribution. Complementary to the more traditional quantitative approach to cognitive evaluation and interpretation, which focuses primarily on "how much" is achieved on a cognitive task (e.g., how many words can be recalled or how many pictures can be named), a more qualitative, process oriented, approach to neuropsychological evaluation emphasizes the careful observation of "how" a cognitive task is performed, the kind of cognitive processes and strategies that are employed en route to solving the task-whether it is passed or failed-and the quality of the errors made in the execution of the task. Beyond a pure analysis of a single achievement score, this approach affords the examiner rich and clinically relevant information to answer the fundamental question of "why" a person fails on a given task, helping to link the nature of the primary cognitive deficits affecting test performance with the underlying brain dysfunction and, therefore, informing diagnostic conclusions.

The process-based approach (PBA) to neuropsychological evaluation was mainly used in Europe up until the early 1970 s, informed predominantly by the outstanding contribution of Alexandre Luria (Luria, 1980; Poreh, 2000, 2006) and, in America, led by the pioneering work of Edith Kaplan $(1988,1990)$ and her working group at the Clinical Neuropsychological Services at the Boston Veterans Administration Medical Center. Efforts were made to adapt the process approach for use with traditional standardized neuropsychological tests and to develop standardized methods of administration and evaluation and analysis of error patterns as well as deriving indices to help capture a host of cognitive functions required for the successful completing of these tests (Ashendorf, Swenson, \& Libon, 2013). Resulting from the revolutionary contributions of Kaplan and her colleagues, this approach has become known as the Boston Process Approach (BPA; Milberg, Hebben, \& Kaplan, 1986).

Informed by the principles and methodology of the BPA, and with the aim of assisting examiners in their interpretation of MoCA test performance and their exploration of the cognitive profile of respondents, we set out in this article to conduct a review of the current psychometric features of the standard MoCA to differentiate throughout the continuum from normal cognitive aging and dementia, and to present a proposal for a PBA version of the standard MoCA, namely MoCA-PA. While this modified version incorporates a number of additional qualitative features designed to help capture the various cognitive functions involved in each task, an effort was made to avoid interfering with the standard administration of the original test, thus allowing clinicians to obtain a total achievement score referable to currently available normative data. Thus, largely, these qualitative modifications involved techniques of data collection and analyses rather than change in the actual test administration itself. With these modifications, we aim to assist clinicians in the process of their interpretation of cognitive test performance and their capacity to identify the strengths and weaknesses of a respondent's cognitive profile. It should, however, be recognized that the MoCA-PA, like the MoCA itself, is not intended, nor should it be treated as, a substitute of a detailed cognitive evaluation.

\section{MoCA: Psychometric properties and clinical utility}

The MoCA is a well-designed and extensively validated cognitive screening tool proven to be clinically useful in differentiating MCI and mild Alzheimer's disease (AD) from normal cognitive aging. The advantages and psychometric properties of the MoCA have often been presented in the 
literature in comparison with the ubiquitous MMSE. In the original validation study, using a cut-point of $<26 / 30$ for impairment, the MoCA showed substantially higher levels of sensitivity (90\% for MCI and $100 \%$ for dementia) compared to the MMSE (18\% for MCI and $90 \%$ for dementia) while maintaining a high level of specificity (87\%). Comparative analysis of subsequent studies using the MoCA, revealed an average sensitivity range of $85 \%$ (range 67-96\%) for MCI and of $94 \%$ (range $88-100 \%$ ) for $\mathrm{AD}$, with an average specificity (i.e., correct identification of healthy controls) of $76 \%$ (range 19-98\%). This variability is thought to reflect differences in selection criteria of healthy controls and demographic variables of participants, variation in setting (community vs. memory clinic), and cultural factors (Julayanont \& Nasreddine, 2017). The greater sensitivity of the MoCA relative to the MMSE has been attributed to its lower ceiling effects, the more challenging nature of its items, in particular memory, and the introduction of higher order attention and executive items (Biundo et al., 2016). In a longitudinal evaluation, the MoCA has also been shown to be more sensitive to cognitive decline in a short period of time (mean 167 days) compared to the MMSE, providing comprehensive information on the profile of clinical deterioration (Freitas, Santana, \& Simoes, 2010).

Age and education has been consistently shown to affect the overall achievement score on the MoCA with some subtests being more sensitive than others to demographic variables. The original validation study recruited highly educated healthy controls ( $13.3 \pm 3.3$ years of education) and recommended a correction of 1 point for those with 10-12 years of education. Subsequent validation studies in clinical samples have consistently shown that using the originally recommended cut-point of $<26$ for impairment results in very high sensitivity rates at the cost of yielding unacceptably low specificity rates (60\% or less) (Davis et al., 2015) and lower cut-points have been recommended, which maximize levels of specificity without compromising sensitivity rates (Coen, Cahill, \& Lawlor, 2011; Freitas, Simoes, Alves, Duro, \& Santana, 2012; Paul et al., 2011; Waldron-Perrine \& Axelrod, 2012). Pendlebury, Mariz, Bull, Mehta, and Rothwell (2012) suggested that optimal cutoffs will depend on whether the MoCA is used for screening (thus requiring a high sensitivity) or diagnosis (requiring a high specificity). For example, in a memory clinic setting, Smith et al. (2007) found that using the index cut-point of $<26$, the MoCA yielded a sensitivity of $83 \%$ for the detection of MCI but only $50 \%$ specificity. Also, in a memory clinic with a population of MCI and Dementia (mean MMSE = 23.6 \pm 3.8 ) and employing the same index cut-point of $<26$, Larner (2012) found that MoCA was more sensitive than MMSE (0.97 vs. 0.65$)$ but less specific ( 0.60 vs. 0.89$)$; however, when adjusting the MoCA cutoff to $\geq 20 / 30$, specificity improved (0.95) at the expense of some loss of sensitivity (0.63). Conversely, in a community dwelling sample Luis et al. (2009) revealed that the index cut-point yielded $97 \%$ sensitivity for the detection of AD and MCI but only $35 \%$ specificity, whereas downward adjustment to 23 exhibited excellent sensitivity (96\%) and specificity (95\%). As stated by Hoops et al. (2009) these findings suggest that a positive screen using MoCA requires additional assessment due to suboptimal specificity at the recommended screening cutoff point.

Population based studies confirm the fact that the original cut-point is too stringent and may lead to the misclassification of normal individuals as impaired (Kenny et al., 2013; Malek-Ahmadi et al., 2015; Rossetti et al., 2011). For example, Rossetti et al. (2011) found that even when correcting for education, $62 \%$ of their population scored below the index cut-point of $<26$ and would fall in the MCI range, which was misleading. These findings underscore the importance of considering demographic factors when interpreting test performance, particularly given the high failure rate on certain items in the general population with most frequently missed items including the cube drawing (59\%), delayed free recall (56\%; $4 / 5$ words), sentence repetition (55\%), placement of clock hands (43\%), abstraction items (40\%), and verbal fluency (38\%; 11 words in $1 \mathrm{~min})$.

Beyond mild dementia and MCI due to AD, the MoCA has also been validated in a wide range of clinical populations including vascular cognitive impairment (both with asymptomatic and symptomatic cerebrovascular disease patients such as post-stroke or TIA, heart failure, and chronic atrial fibrillation), a range of movement disorders such as Parkinson's and Huntington's Disease, space occupying lesions, systemic lupus erythematosus, substance use disorders, idiopathic rapid eye movement sleep behavior disorder and chronic obstructive pulmonary disease, epilepsy, and behavioral variant fronto-temporal dementia, demonstrating greater degree of sensitivity compared to the MMSE (Dagenais et al., 2013; Dong et al., 2010; Julayanont \& Nasreddine, 2017).

The concerns regarding the specificity of the original cutoff score has also been found in these clinical conditions and different optimal cut-points have been recommended. For example, Dalrymple-Alford et al. (2010) found that MoCA cut-point of 21 for dementia and 26 for MCI were the most appropriate when screening cognition in Parkinson's Disease (PD). Moreover, a significantly lower cut-point of $<17$ yielded optimal discriminatory capacity in a population of behavioral variant of Frontotemporal Dementia (Freitas, Simoes, Alves, Duro, et al., 2012) and Vascular Dementia, with a cutoff below 8 on the short version in the latter population (Freitas, Simoes, Alves, Vicente, \& Santana, 2012).

However, despite the advantages of the MoCA over the MMSE, a number of disadvantages have been listed. For example, Cordell et al. (2013) argued that studies evaluating the utility of the MoCA in general practice settings are lacking, the administration time is over 10 minutes and it has an inherent education bias limited use. Regarding the effect of demographic variables and in particular the impact of education, Malek-Ahmadi et al. (2015) suggested that age- and education-corrected normative scores may provide a more refined estimate of cognitive performance and may reduce the occurrence of misclassification at screening, although Gagnon et al. (2013) noted that adjusting the MoCA total 
score for education had a detrimental effect on sensitivity with only a slight increase in specificity. For Hanagasi et al. (2011), neither MoCA nor other screening tools provide a satisfactory combined sensitivity and specificity levels for the diagnosis of PD-MCI.

Recommendations for increasing the clinical utility of the MoCA have included weighting its subtests according to their respective diagnostic values (Fengler et al., 2016). Horton et al. (2015) suggested that some MoCA items do not add appreciably to the clinical sensitivity of the standard version and that similar classification rates can be achieved with an abbreviated version. Rossetti et al. (2011) found that some MoCA items were useful in detecting cognitive impairment, while other items were rarely missed and lacked sensitivity. In relation to this, Mai et al. (2013) found that a mini-MoCA comprising the CDT, five-word delayed recall, and abstraction represents a potential alternative to the full MoCA in patients with cerebrovascular disease, since a score of $<7 / 10$ using this 10 -point mini-MoCA identified cognitive impairment as defined by the MoCA with a sensitivity of $98.5 \%$, and a specificity of $77.6 \%$. Finally, Cecato, Martinelli, Izbicki, Yassuda, and Aprahamian (2016) found that, taken as individual items, word repetition, digits backward, serial 7, phrases, verbal fluency, abstraction, and word recall discriminated between $\mathrm{MCI}$ and healthy control participants $(p<0.001)$, and that the clock drawing, the rhino naming, delayed recall of five words, and orientation discriminated between patients with MCI and $\mathrm{AD}(p<0.001)$. However, while it remained unclear that all the MoCA subtests are needed to identify cognitive impairment, a reduced version of the MoCA with only a specific series of items did not improve accuracy between MCI and healthy controls $(p=0.076)$ or MCI and AD $(p=0.119)$.

This consideration of MoCA items separately also requires some considerations regarding the existence of variants to the original standard administration. A total of three alternative versions of the MoCA exist in English (with different words for episodic memory and different pictures for Confrontational Naming), with reported comparable reliability to the original (Costa et al., 2012). However, the authors recognize that the appropriate cutoff value seems to be population-specific (Waldron-Perrine \& Axelrod, 2012). Therefore psychometric measures such as discriminative validity, test-retest reliability, and the use of population based norms, require further research for alternate forms. There is also an alternate version for the visually impaired, the MoCA-Blind (Wittich, Phillips, Nasreddine, \& Chertkow, 2010), where the Trail Making Test (TMT), the copy cube task, the CDT, and confrontational naming were removed. This led to a maximum score of 22 for the total MoCABlind. Instead of merely subtracting 8 points to the total score (i.e., from 25 to 17, which seriously compromised and reduced the sensitivity to 0.44 for $\mathrm{MCI}$ and 0.87 for $\mathrm{AD}$ ), the relative shift was calculated, whereby the failure point was moved in proportion to the total score, thus leading to a cutoff score of 18 out of 22 (which still reduced the sensitivity to 0.63 for MCI but maintained a good rate of 0.94 for $\mathrm{AD})$. Thus, authors recommended using this version of the test with caution until further improvements are made to increase its sensitivity to higher than $80 \%$ when it is used without the items that require functional vision. Also, the MoCA-Basic for low educated or illiterate subjects (Julayanont et al., 2015) provided a $81 \%$ sensitivity and $86 \%$ specificity in screening of MCI with accuracy of $84 \%$ in a small community hospital sample in Thailand, but still requires further research to see how it can be applied to culturally different larger clinical samples. Moreover, Roalf et al. (2016) developed a short version of the test, the sMoCA, which identified 8 MoCA items with high item discrimination, high correlation with the original MoCA, and was useful for clinical settings with high time constraints (as it takes only $5 \mathrm{~min}$ to administer).

Moreover, individual MoCA items and domain subgroups yield insufficient information to draw conclusions about impairment in specific cognitive domains, as we will see in the following section.

\section{Brief review of the MoCA cognitive tasks: What do they actually measure?}

Like many other cognitive screening instruments used in the detection of MCI and dementia, the MoCA represents an amalgamation of commonly used neuropsychological tests (e.g., TMT, CDT, Confrontational Naming, List Learning and List Recall, Digit Span, Serial 7s, Sentence Repetition, Phonetic Fluency, Similarities, and Orientation to time and place), most of which have been shortened or simplified for speed and ease of administration and, in some cases, the primary outcome variable has been modified.

For example, the MoCA employs a very brief confrontational naming task, resembling the original and lengthier (60 items) Boston Naming Test (BNT) (Goodglass \& Kaplan, 1983). In the MoCA's naming task, the examinee is asked to name three animals and a binary score of $0-1$ (pass/fail) is used to measure correct and incorrect responses to each item. Scores on the 3 items are then added to derive a total achievement score. However, unlike the BNT where, in the case that a respondent fails to name an object, a predetermined semantic cue (e.g., an ocean animal for octopus) is provided and, if the respondent fails to be assisted by this cue, a phonemic cue consisting of the initial sound of the target word is provided, the MoCA naming test does not include this administration protocol. We believe that complementing the test with this "satellite" cueing system would result in minimal additional time of administration and yet be useful in helping to isolate the primary underlying cognitive mechanism responsible for an incorrect response.

In other cases, the MoCA employs a shortened version of the standard neuropsychological test and modifies its original primary outcome variable. This is the case for TMT Part B (Reitan \& Wolfson, 1992), a complex task requiring the capacity to establish and maintain a complex mental set. Unlike the original task, which employs time to complete the task as the primary outcome variable, the MoCA Trail Making uses the presence of uncorrected errors as an indication of failure on the task. 
Additionally, given the complexity of these tasks, there is often a minimal amount of agreement regarding the operational definition of the cognitive construct and the precise cognitive domains that these tests are purported to measure (Coen et al., 2016; Kaufman, Boxer, \& Bilder, 2013). A recent study, employing an Exploratory Factor Analysis, showed that many MoCA items cross-load onto several factors and do not necessarily measure any single domain (Coen et al., 2016). For example, the usage of a shortened version of the original Controlled Oral Word Association Test (COWAT; Benton \& Hamsher, 1976), with the sole administration of the letter $F$ and asking respondents to generate as many words beginning with this letter, places this task under the subheading of language. While undoubtedly this complex task requires a range of language skills, such as lexical retrieval, it also places demands on a range of higher order executive functioning skills such as strategic search, sustained attention, response inhibition, and cognitive flexibility and, in fact, it is most often employed by neuropsychologists as a measure of executive function (Henry, Crawford, \& Phillips, 2004).

For all these reasons, the development of a PBA version of the MoCA that could examine patients' performance beyond the discussion of total scores, cutoff points, and specific groups of items may be a worthwhile approach to improve the clinical utility of the MoCA for the screening of MCI and dementia.

\section{Proposal of a Process-Based Approach version of the MoCA}

In order to develop the MoCA PA version (MoCA-PA), we drew from the work of different authors who have already shown how the analysis of errors conveys additional information regarding underlying brain/behavior relations (e.g., TMT or verbal fluency). We took inspiration from the previous work by Price et al. (2011), who had already indicated the benefits of the analysis of errors elicited on the CDT drawing in the MoCA subtest; and the benefits of adding a copy condition to help identify the primary underlying cognitive deficits responsible for errors in the command condition. We also drew from the work of Hodges, Salmon, and Butters (1991) on the error analysis of the BNT, proving to be effective beyond the overall achievement score in distinguishing between Huntington's disease and $\mathrm{AD}$. Furthermore, the analysis of errors produced on the fluency task of the MoCA-PA is inspired by the work of Troyer, Moscovitch, and Winocur (1997).

The MoCA-PA retains the original quantitative 30-point scoring system with minor changes to the standardized administration that we believe do not alter the nature or complexity of the tasks themselves. Efforts have been made to keep the administration and scoring form in one sheet, which is now a two-sided page. However, due to space limitations, an additional scoring sheet for the record and analysis of the newly introduced qualitative indices has been developed.

While the MoCA-PA retains the majority of actual administration format intact, it does introduce some minor changes as outlined in the this section in order to help capture important qualitative aspect of test performance. For example, the instructions are now embedded in the test record form itself to ensure fidelity to administration procedures and to avoid truncating the cognitive demands of the task. In this vein, Coen et al. (2011) warned that failure to adhere to the test instructions of the memory subtest can turn the new learning section of the task into a span of apprehension task unless the respondents are told that they must remember the words both now and at the end of the test. To facilitate the analysis of errors, adequate space is now provided to record verbatim the respondents' answers. Minimal modifications to test instructions (i.e., Naming tasks: respondents are not told that the pictures are animals) were introduced. Lastly, minimal changes were introduced in the way that responses are recorded. For example, in the learning condition of the memory tasks, examiners are asked to record the serial order of word recall by numbering the responses in the order of production on both trials and to record verbatim all instances of intrusion errors. Inclusion of these modifications gives rise to a marginal increase in total test administration time, extending it to a maximum of $15 \mathrm{~min}$. In fact, while our default option is to administer the complementary tasks in the sequential order of the existing subtests (e.g., CDT Command followed by Copy condition; Letter Fluency followed by Semantic Category Fluency, etc.), the user may wish to implement these modifications at the end of the standard test. Although for the purpose of subsequent analysis, the recording of verbatim responses and type of errors, as proposed here, must be carried out during the test administration.

Informed by the methodological framework of the PA, we employ two main methodologies (Poreh, 2000) in order to capture the underlying cognitive deficits responsible for test underperformance:

- The "Satellite" Testing Paradigm: This approach consists of the inclusion of complimentary conditions to the existing cognitive task with the aim of isolating the individual cognitive processes necessary for its completion. Such a paradigm assists in the identification of the precise nature of the cognitive deficit responsible for test performance on the original cognitive task. Using this paradigm, satellite conditions for the CDT (e.g., copy and tracing conditions) and the Naming Task (i.e., semantic and phonetic cue) were introduced.

- The Composition Paradigm: This approach consists of the generation of new indices in an already existing cognitive task, using the data that has already been derived from the standardized administration of this task but not previously analyzed. New indices were derived for the verbal fluency tests (i.e., switching and clustering) and memory tasks (e.g., gained and lost access, etc.). A series of qualitative classifications of errors were introduced for a series of tasks including TMT, CDT, verbal fluency, and memory.

\section{MoCA-PA: Suggested modifications for each cognitive task}

Details of all the modifications made in constructing the MoCA-PA are provided as Supplementary Materials. 
However, by way of example and in order to aid understanding of the types of changes made, the modifications implemented in TMT, CDT, and verbal fluency are detailed here.

\section{MoCA-PA: Alternating Trail Making}

Like any other cognitive test, the TMT condition used in the original MoCA, is a complex cognitive task requiring multidimensional cognitive skills some of them of a lower order, such as numerical and alphabetical knowledge required for sequencing, visual scanning, and processing speed and others of a higher order, including the ability to sustain attention and switch flexibly between two cognitive sets (Delis, Kaplan, \& Kramer, 2001).

The original MoCA employs the number of errors made in the task as the single achievement score to quantify test performance. Following the composition paradigm of BPA's methodology (Poreh, 2000), the MoCA-PA analyzes the nature of errors made during the standard administration of the task in order to assist in the determination of the nature of the precise or at least primary cognitive deficit responsible for failure. Hence, for MoCA-PA version, the Alternating TMT may register the following error subtypes (based on Ashendorf et al., 2013), requiring the examiner to record the nature of the error/s that were made by the test taker, whether these were immediately self-corrected or undetected:

- A sequencing error: This occurs when the alternating set is maintained but the incorrect target is selected from the proper sequence (e.g., 1-A-2-B-4 or 1-A-2-C). Sequencing errors are typically considered to be a result of working memory interference resulting from difficulty with sequential management of the two components of the alternating series. While this error may be the result of poor mastery of the alphabet, this typically only occurs beyond the letter G (Ashendorf, 2013).

- A set-loss error: This occurs when the complex alternating set is lost and the individual proceeds from a number to another number or from a letter to another letter. Set loss errors are considered to represent a failure of cognitive flexibility or difficulty adhering to or recalling the tasks directions (Ashendorf, 2013).

\section{MoCA-PA: Visuoconstructional skills (Clock)}

The CDT is a widely used brief tool to detect cognitive decline associated with a variety of neurobehavioral disorders (Ahmed et al., 2016); hence, it has proved useful in dementia assessment and for assessing numerous cognitive functions. The Command-condition of the CDT, which requires the participant to draw a clock from memory on a blank sheet of paper, places high demand on visual memory and conceptualization, language skills, and verbal memory. However, as Price et al. (2011) state, drawing a clock to the command condition alone cannot effectively distinguish dementia individuals from controls, or even between dementia subgroups. They found that diagnostic differences occurred only when command and copy performances were compared. The command-copy discrepancy is consequently useful for assessing patients' cognitive strengths and weaknesses and assisting with preliminary differential diagnosis.

Different approaches, administration and scoring systems have been used, but one of the scoring systems with the largest acceptance for the qualitative analysis of errors is the one developed by Rouleau, Salmon, Butters, Kennedy, and McGuire (1992), which included assessment of clock size, graphic quality, pull to stimulus, conceptual difficulty, spatial organization, and perseverative responses. This scoring system was subsequently reviewed and quantified by Cosentino et al. (2004). Taking the best of both approaches, we present a proposal for administration and scoring that adds two new conditions and a simplified scoring method that aims to assess qualitative aspects of performance while helping to uncover the underlying cognitive deficits associated to that performance.

The CDT command condition requires comprehension of verbal instructions, access to semantic attributes associated to the "clock," the ability to syntactically process "ten past eleven," the retrieval of a visuoperceptual representation of a clock, and mental planning (with regard to the details of the figure-spacing, aligning, hand length).

The copy condition requires the involvement of visuoperceptive and visuospatial aspects of the model the test taker has to copy and planning with regard to initiating and completing the motor work. Thus, comparing performance between both test conditions (copy vs. command) may provide useful information for differential diagnosis, as those patients with predominant semantic deficits and conceptual errors will fail more likely in the command condition (e.g., AD) while those with dysexecutive syndrome or graphomotor problems will make more errors in copy condition (e.g., Huntington's Disease). In addition, to rule out any other error that may mask the graphomotor performance, a third condition, the tracing condition, will be included (examinee will be provided with the same clock from the copy condition drawn as a watermark, and is instructed to follow the trace and complete the clock drawing on the watermark), based on the previous work by Evans, Coen, Burke, and Lawlor (2005).

For the MoCA-PA, in addition to the traditional single score, new qualitative indices have been introduced, following both the satellite (with the inclusion of a new task, i.e., tracing) and the composition paradigm of Quantified Process Approach methodology (Poreh, 2000):

- Gross Motor Errors: Graphomotor difficulties:

- Distortion of the clock face (disparity between the vertical and horizontal diameters, or draws a different shape-square).

- Hands not straight. 
- Hands do not connect in the middle. Anchor not located in the middle of the clock.

- No size difference between the hands.

- Segmentation: Stroke interruptions, broken lines.

- Multiple attempts (i.e., draws more than one circumference, or attempts to do it).

- Stimulus-bound response: Tendency of the drawing to be dominated or guided by a single stimulus.

- Hands are set for 10 to 11 rather than 10 past 11 (specificity of 0.96 for Alzheimer's Dementia) (Ryan et al., 1995).

- Pull to Stimulus (in the copy condition, a tendency to draw over the "model").

- Closing-in (Rouleau, Salmon, \& Butters, 1996): in the copy condition, a tendency to draw over the lines of the model and/or trace the lines from the model to the surrounding space).

- Conceptual deficit: Conceptual errors reflect a loss or a deficit in accessing knowledge of the attributes, features and meaning of a clock.

- Misrepresentation of the clock itself:

- Misrepresentation of the time of the clock.

- Absence of circumference.

- Numbers missing, absence, or poor representation of numbers.

- Only one hand or no hands.

- Dots instead of numbers (Freedman et al., 1994).

- Uses letters or numbers to write the time (Rouleau et al., 1992).

- Spatial and/or planning deficit: Deficit in the layout of numbers on the clock:

- Neglect of the left hemisphere (numbers placed in the right half of the clock).

- Deficit in planning, leaving a gap before "12," “3," "6," or "9," depending on the strategy used in drawing.

- Numbers outside the clock face.

- Numbers placed counterclockwise.

- Circular format: ability to maintain the appropriate circular format when placing the numbers in relation to the clock face.

- Perseveration:

- Perseveration of hands/Cart wheel phenomenon (Cacho, García-García, Arcaya, Vicente, \& Lantada, 1999).

- Hyperkinetic perseverations (participant unable to desist from executing a graphomotor response once the response has been completed).

- Perseveration of numbers or interminable perseverations (e.g., the patient writes the number 6 repeatedly).

- Additional features:

- Performance comparison between command, copy, and tracing condition.

- Total time for completion.

- Time elapsed from writing the last number and setting the first hand.

- Total time to complete the hand setting.

\section{MoCA-PA: Verbal fluency}

The verbal fluency task requires the participant to generate lexical items while simultaneously observing rules or restrictions, such as (1) the words generated must share the same initial orthographic property, and (2) names of people or places, numbers, or grammatical variants of previous responses are not allowed. It represents a relatively novel and complex task that imposes comparable demands upon executive or supervisory processes because both require efficient organization of verbal retrieval and recall, as well as self-monitoring aspects of cognition (tracking of previous responses), effortful self-initiation (to generate required answers), simultaneous processing (observing the multiple rules of the task), systematic retrieval of phonemically similar lexical items, speed of processing, and inhibition of responses when appropriate (to avoid words that instructions do not consider correct, like names of people or places, etc., as previously stated). Moreover, phonemic fluency requires the creation of search strategies based primarily on lexical representations, as well as fundamental cognitive components including vocabulary knowledge, spelling ability, and attention (Henry et al., 2004).

However, according to a recent study by Vaughan, Coen, Kenny, and Lawlor (2016), the inclusion of a semantic fluency task that serves as a comparison with phonemic fluency may add significant value to the screening for MCI and dementia, and can be quickly added to any assessment protocol. This study found that the semantic advantage (i.e., better performance for animal vs. letter $\mathrm{F}$ fluency) persists into later life in a population-based sample of communitydwelling older adults, and that this pattern is reversed in Alzheimer's dementia (i.e., loss of semantic advantage in $\mathrm{AD}$, yielding a phonemic advantage). Hence, the inclusion of both types of fluency tasks and the comparison of their performance (in the form of discrepancy scores between phonemic and semantic fluency, for example) can help distinguish between normal and abnormal cognitive aging

Based on this, for the MoCA-PA, an additional semantic condition (i.e., animals) was included and new error indices are introduced (Troyer, 2000). To capture cognitive processes of initiation of verbal response and capacity to sustain response and mental effort over time, responses are recorded verbatim for each 15" segment; and two types of errors are scored: set-loss and repetition. A complementary semantic task is introduced and two qualitative indices are recorded: clustering and switching.

In summary, the added indices are presented as follows:

- Discrepancy between phonemic and semantic fluency.

- Number of words generated within each of the four $15 \mathrm{~s}$ time intervals.

- Set loss errors:

- Due to poor spelling (e.g., phone, phobia for "F" letter).

- Set loss errors: words starting with other letters.

- Repetition errors:

- With temporal proximity (reflects perseveration, inability to inhibit a previous response).

- With temporal distance (reflects memory problem). 


\section{Pilot study}

\section{Goals}

Once the modified version was developed, the initial goal was (1) to develop a pilot study of the MoCA-PA with older individuals living in the community; (2) to compare data obtained when relying on traditional scoring methods (total scores alone) and the information provided through analysis of test takers' strategies and responses (processes); and (3) to identify potential useful indices for further consideration in future larger sample studies. Knowing in advance that such a small sample size will involve limitations and necessary caution in generalizability of results, we tried to focus on both quantitative and qualitative aspects of the information obtained.

\section{Sample and methods}

The sample was composed by 45 community-dwelling older individuals (16 male, 29 female; mean age $=71.13$, $S D=5.94$ ), attending a University program for older people in Spain. The consent from participants was obtained on a written basis and data were analyzed anonymously. A third of the sample $(33.3 \%)$ had completed primary studies, while $42.9 \%$ had finished secondary and $23.8 \%$ had developed third level university studies. None of them had a recognized clinical cognitive decline. All of them were administered the MoCA-PA version (Blanco-Campal et al., 2016). All the statistical analysis were performed using SPSS version 23.0 for Windows.

\section{Results}

\section{Descriptive results}

According to the original MoCA instructions, those with less than 12 years of education were added 1 point to the total score. In the Spanish norms by Ojeda et al. (2016), suggested cutoff scores by age are 26 points for the age range from 56 to 65,24 points from 66 to 70 years-old, 22 points from 71 to 75 years-old, 21 points from 76 to 80 years-old, and 19 from 81 to 86 years old. According to this, $44.4 \%$ of our sample is below the cut-point (mean$=22.69, S D=3.92$, range: $14-30$ ).

In terms of specific subtest performance, Table 1 shows the time for completion of the TMT and the three variants (command, copy, tracing) of the CDT, where scores show a great heterogeneity of performance among the participants, but overall similar completion time for the command and copy variants of the clock.

Table 1. Time to complete TMT and clock drawing variants (in seconds).

\begin{tabular}{lccc}
\hline Test & Mean & SD & Minimum-maximum time \\
\hline TMT & 28.48 & 20.40 & $6.80-102.70$ \\
Clock Drawing (Command) & 44.11 & 23.04 & $15.00-122.70$ \\
Clock Drawing (Copy) & 43.67 & 25.21 & $12.40-125.08$ \\
Clock-Drawing (Tracing) & 57.86 & 27.26 & $20.20-181.70$ \\
\hline
\end{tabular}

Note. TMT $=$ Trail Making Test. $n=45$.
In terms of errors, $26.7 \%$ of the sample made errors in the TMT, $40 \%$ in the Cube Copy, $48.9 \%$ in the Command CDT, $31.1 \%$ in the Copy CDT, and only $2.2 \%$ in the Tracing CDT. In TMT, set-loss errors (alone or in combination with repetition errors) are the most prominent. Command CDT shows a majority of conceptual and planning errors (combined 36.6\%), with less than a quarter of participants having anchored the clock numbers as a strategy for task completion. Copy CDT shows a majority of gross motor errors, with more than a quarter of participants using an anchoring strategy. Tracing CDT only shows a single gross motor error for one participant. For the naming test, $2.2 \%$ failed in naming the lion, $28.9 \%$ failed in naming the rhinoceros, and nobody failed item 3 (dromedary/ camel). In the Concentration task, $13.3 \%$ of the sample made errors. Table 2 goes into more detail in terms of the specific types of errors committed in all these tests.

A detailed analysis of errors and word count in both phonetic and semantic fluency can be seen in Table 3 . It is observed that $66.7 \%$ of the participants made some kind of error in phonetic fluency, and 60\% made errors in phonetic fluency. In both cases, set-loss errors (alone or in combination with repetition errors) are the most prominent and the majority of words are produced in the first $15 \mathrm{~s}$ interval (e.g., from 0 to $15 \mathrm{sec}$ ).

Table 4 shows performance in Digit Span Forward and Backward differentiating between the actual span (i.e., correct digits produced in any order) and the serial order. As it can be seen, performance differs when considering only serial order as per original MoCA instructions and considering both scores as part of different cognitive processes.

Table 5 presents the process analysis for Abstraction items. With $40 \%$ making some type of error for the trainbicycle item and $57.8 \%$ for the watch-ruler item, the majority of errors are of the in-set subordinate type (i.e., describing a common concrete feature in terms of comparing each pair of items in a more abstract way, e.g., "both have wheels," "both have numbers" ...).

Finally, Table 6 presents performance for memory subtests, which shows a clear better performance for immediate recall when compared to free delayed recall.

\section{Age and gender effects}

Due to the lack of normal distribution of scores in the MoCA-PA, we performed a Spearman correlation analysis to see how age was related to performance. Scores showed an inverse correlation between age and total words in both phonetic (rho $=-0.393, p=0.08$ ) and semantic fluency (rho $=-0.362, p=0.015$ ); age and total digits backwards in serial order (rho $=-0.360, p=0.015$ ); and age and gained access (i.e., difference between free and immediate recall) (rho $=-0.433, p=0.003$ ). The older the participants, the worse their performance in some tasks involving executive functioning.

With regards to gender, a Mann Whitney $U$ test performed to analyze differences between male and female showed a significant better performance of female participants in memory related scores: MoCA Memory Index $(m$ 
male 17.31 vs. $m$ female $26.14 ; p=0.030)$, Immediate Recall ( $m$ male 19.41 vs. $m$ female $24.98 ; p=0.030)$, Free Recall $(m$ male 15.88 vs. $m$ female 26.93; $p=0.006)$, and Gained Access ( $m$ male 17.06 vs. $m$ female 26.28; $p=0.022$ ).

\section{Anchoring}

To establish whether anchoring numbers was a useful strategy for performance, a Mann Whitney $U$ test was performed to compare those who anchored with those who did not in many of the numerical scores (e.g., TMT and CDT variants completion times, immediate and delayed memory, digit span, both ANY order and SERIAL order and gained access). There were no statistically significant differences for any of these tasks between those who anchored and those who did not. Subsequently, for the analysis of interaction between anchoring and error commission in different subtests as dichotomic variables, a chi-square test of independence was performed. Results showed a statistically significant interaction between anchoring and errors performed in the Command version of CDT $\left(\chi^{2}=8.282\right.$, $p=0.004)$, evidencing anchoring as an effective strategy for

Table 4. Digit span (forward and backwards) any order versus serial order.

\begin{tabular}{llcc}
\hline Test & Mean & SD & $\begin{array}{c}\text { Minimum-maximum } \\
\text { digit count }\end{array}$ \\
\hline Digit Span Forward: any order & 9.87 & 0.405 & $8-10$ \\
Digit Span Forward: serial order & 9.04 & 1.700 & $3-10$ \\
Digit Span Backward: any order & 10.67 & 0.640 & $8-11$ \\
Digit Span Backward: serial order & 8.60 & 2.380 & $3-11$ \\
\hline
\end{tabular}

Note. $n=45$.

Table 2. Process analysis in graphomotor tests, naming and concentration

\begin{tabular}{|c|c|c|c|}
\hline Test & Type of error & Cases & Percentage \\
\hline \multirow[t]{3}{*}{ Trail Making Test } & Set loss errors only & 5 & 11.1 \\
\hline & Sequence errors only & 3 & 6.7 \\
\hline & Both set loss and sequence errors & 4 & 8.9 \\
\hline Cube & Incorrect lines & 18 & 40.0 \\
\hline \multirow[t]{6}{*}{ Clock Drawing (Command) } & Gross Motor & 10 & 22.2 \\
\hline & Linked to Stimulus & 1 & 2.2 \\
\hline & Conceptual & 8 & 17.8 \\
\hline & Planning & 8 & 17.8 \\
\hline & Perseveration & 2 & 4.4 \\
\hline & Anchoring & 10 & 22.2 \\
\hline \multirow[t]{6}{*}{ Clock Drawing (Copy) } & Gross Motor & 8 & 17.8 \\
\hline & Linked to Stimulus & 0 & 0 \\
\hline & Conceptual & 3 & 6.7 \\
\hline & Planning & 5 & 11.1 \\
\hline & Perseveration & 3 & 6.7 \\
\hline & Anchoring & 12 & 26.7 \\
\hline Clock Drawing (Tracing) & Gross Motor & 1 & 2.2 \\
\hline Naming (Lion) & Ambiguous/visual semantic error & 1 & 2.2 \\
\hline \multirow[t]{5}{*}{ Naming (Rhinocerus) } & Visual error & 9 & 20.0 \\
\hline & Ambiguous visual/semantic category error & 1 & 2.2 \\
\hline & Semantic (within category) error & 1 & 2.2 \\
\hline & Unrelated error & 1 & 2.2 \\
\hline & Don't know & 1 & 2.2 \\
\hline \multirow[t]{2}{*}{ Concentration } & Omissions & 5 & 11.1 \\
\hline & Commissions & 1 & 2.2 \\
\hline
\end{tabular}

Note. $n=45$.

Table 3. Error analysis and word count in phonetic and semantic fluency.

\begin{tabular}{llcc}
\hline Test & \multicolumn{1}{c}{ Type of error } & Cases & Percentage \\
\hline Phonetic fluency & Set loss errors only & 17 & 37.8 \\
& Repetition errors only & 2.4 & 24.4 \\
& Both set loss and repetition errors & 11 & 40 \\
Semantic fluency & Set loss errors only & 18 & 8.9 \\
& Repetition errors only & 4 & 11.1 \\
Test & Both set loss and repetition errors & 5 & Min-Max. word count \\
Phonetic fluency (0-15s) & Mean & $5 D$ & $2-10$ \\
Phonetic fluency (16-30s) & 5.71 & 1.69 & $1-7$ \\
Phonetic fluency (31-45s) & 3.76 & 1.57 & $0-6$ \\
Phonetic fluency (46-60s) & 2.29 & 1.60 & $0-6$ \\
Phonetic fluency (Total words) & 2.18 & 4.72 & $5-24$ \\
Semantic fluency (0-15s) & 13.93 & 2.03 & $3-11$ \\
Semantic fluency (16-30s) & 7.04 & 1.83 & $0-8$ \\
Semantic fluency (31-45s) & 4.13 & 1.80 & $0-7$ \\
Semantic fluency (46-60s) & 3.13 & 1.82 & $0-7$ \\
Semantic fluency (Total words) & 2.51 & 4.70 & $6-27$ \\
Gained fluency & 16.82 & 4.99 & $-8-14$ \\
$\quad$ (Semantic - Phonetic Fluency) & 2.89 & & 0 \\
\hline
\end{tabular}

Note. $n=45$. 
Table 5. Process analysis in abstract tasks.

\begin{tabular}{llrr}
\hline Test & \multicolumn{1}{c}{ Type of answer } & Cases & Percentage \\
\hline Train-Bicycle & 2-point answer & 27 & 60.0 \\
& 0-point: in-set vague & 7 & 15.6 \\
& 0-point: in-set subordinate & 10 & 22.2 \\
& 0-point: out-of-set juxtaposition & 1 & 2.2 \\
Watch-Rule & 2-point answer & 26 & 42.2 \\
& 0-point: in-set vague & 2 & 4.4 \\
& 0-point: in-set subordinate & 13 & 28.9 \\
& 0-point: out-of-set different & 2 & 4.4 \\
& 0-point: other unrelated & 1 & 2.2 \\
& 0-point: other - do not know & 1 & 2.2 \\
\hline
\end{tabular}

Table 6. Analysis of memory task.

\begin{tabular}{lrcc}
\hline Test & Mean & SD & $\begin{array}{c}\text { Minimum-maximum } \\
\text { word count }\end{array}$ \\
\hline Memory: Immediate & 4.82 & 0.44 & $3-5$ \\
Memory: Free Delayed Recall & 2.04 & 1.83 & $0-5$ \\
Memory: Gained Access & -2.78 & 1.72 & $-5-0$ \\
MoCA Memory Index & 9.84 & 3.71 & $2-15$ \\
\hline
\end{tabular}

the CDT (21 out of 22 participants doing errors in the command version had not anchored the numbers). Additionally, another statistically significant interaction was found between anchoring the clock numbers and naming the 'rhinoceros' $\left(\chi^{2}=5.223, p=0.022\right)$, that is, none of the individuals who made naming errors for 'rhinoceros' had anchored the numbers in the Command CDT.

Digit span: Different processes for span in 'any order' versus 'serial order'

Following Lamar, Rhodes, Wambach, Swenson, and Libon (2013) work describing span in any order and span in serial order as tasks with distinct underlying cognitive processes, we performed a Wilcoxon test to analyze the differences in our group of participants between their performance in both Digit Forward and Backward considering performance shown in digits produced in ANY order versus those produced in SERIAL order. Results showed a significant better performance for digits recalled in ANY order, both in digit forward $(z=-3.319, \quad p=0.001)$ and digit backward $(z=-4.593, p=0.000)$.

\section{Verbal fluency: Comparison between phonetic and seman- tic variants}

Following Vaughan et al. (2016) work, we added a semantic category fluency task as a subtest in the MoCA-PA, and following previous PBA studies, we divided the performance registration in $15 \mathrm{~s}$ intervals. We then performed a Wilcoxon test to analyze the differences in our group of participants between their total and partial (for each $15 \mathrm{sec}$ interval) performance in both subtests. Results showed a significant better performance in Semantic Fluency total words relative to Phonemic Fluency $(z=-3.489, p=0.000)$ and also for two intervals, the 0 to 15 seconds interval $(z=-3.869, p=0.000)$ and the 31 to 45 seconds interval $(z=-2.373, p=0.018)$.

\section{Abstraction as a relevant subtest}

For further analysis, participants were divided in two sets of groups: (a) those who made errors in the train-bicycle item versus those who did not, and (b) those who made errors in the watch-ruler item versus those who did not. For the train-bicycle item, those who made errors showed a significant poorer performance in MoCA total score. Mann Whitney $U$ test analyses were performed to check for differences between those performing with and without errors. Results showed significantly worse performance for those who made errors in the train-bicycle item for the MoCA total score $(m$ no errors $=26.62 \quad$ vs. $\quad$ errors $=18.05$, $z=-2.172, \quad p=0.030)$, for the TMT time ( $m$ no errors $=17.60$ vs. $\quad$ errors $=28.95, \quad z=-2.903, \quad p=0.004$ ), phonetic fluency total words ( $m$ no errors $=26.31$ vs. errors $=18.47, z=-1.981, p=0.048)$, and phonetic fluency 31 to $45 \mathrm{~s}$ interval ( $m$ no errors $=26.29$ vs. errors $=18.50$, $z=-2.000, p=0.045)$. For the watch-ruler item, the only significant worse performance for those who made errors was found for semantic fluency total words $(z=-2.032, p=0.042)$.

\section{Immediate versus delayed recall}

We performed a Wilcoxon test to analyze the differences in our group of participants between delayed free recall and immediate recall, as most participants seem to decrease their performance in the delayed recall. Results showed a significant decrease in performance for delayed free recall when compared to immediate recall $(z=-5.474, p=0.000)$.

\section{MoCA cut-point and differences in performance}

We proceeded to dichotomize some of the process performance variables, and then we performed a chi-square test of independence to check whether being above or below the cut-point relates with particular processes, errors, or performance indicators. Results indicated that being above or below the cut-point only related with the occurrence of errors in the "train-bicycle" item of the abstraction task $\left(\chi^{2}=4.664, p=0.031\right)$, with a $63 \%$ of participants with a total below the MoCA cut-point showing errors in that item.

For those variables that were not dichotomous, we performed a Mann-Whitney $U$ test to check the differences between those below and above the MoCA cut-point. Results showed a significant poorer performance for those below the cut-point in the MoCA memory index ( $m$ above$=29.22$ vs. $m$ below $=15.23 ; z=-3.570, p=0.000$ ), completion time for TMT ( $m$ above $=17.67$ vs. $m$ below $=28.30$; $z=-2.734, p=0.006$ ), CDT Copy ( $m$ above $=18.00$ vs. $m$ below $=29.25 ; \quad z=-2.855, \quad p=0.004)$, CDT Tracing $(m$ above $=18.83$ vs. $m$ below $=26.90 ; z=-2.074, p=0.038$ ); total words in phonetic fluency ( $m$ above $=28.48$ vs. $m$ below $=16.15 ; z=-3.137, p=0.002$ ), as well for the 0 to $15 \mathrm{~s}$ interval words ( $m$ above $=27.22$ vs. $m$ below $=17.73$; $z=-2.482, p=0.013)$, the 16 to $30 \mathrm{~s}$ interval words ( $m$ above $=27.14$ vs $m$ below $=17.83 ; z=-2.409, p=0.016)$, and the 46 to $60 \mathrm{~s}$ interval words ( $m$ above $=29.02$ vs. $m$ 
below $=15.48 ; z=-3.494, p=0.000) ;$ concentration commission errors $(m$ above $=20.24$ vs. $\mathrm{m}$ below $=26.45$; $z=-2.095, p=0.036$ ) and gained access ( $m$ above $=27.88$ vs. $\mathrm{m}$ below $=16.90 ; z=-2.831, p=0.005)$. As it can be seen, consideration of each of these test performance indices provides additional relevant data which may help reveal the primary underlying cognitive deficits involved beyond the mere consideration of cut-points.

\section{Discussion}

In this article, we reviewed the current psychometric properties of the standard MoCA and present the proposal of a modified version of standard MoCA, informed by the methodology of the Process Based Approach of neuropsychological evaluation, referred to as the MoCA- Process Approach (MoCA-PA). In developing this qualitative version, our aim was to improve upon the well-established clinical utility of the standard MoCA by incorporating a number of qualitative methods of test administration, observation of the process involved in the execution of each subtest and analysis of error types committed, in order to enhance the scope and depth of the neuropsychological interpretation of cognitive test performance to explore the distinct cognitive profile of respondents. In the preliminary set of data obtained in the pilot study, we found that the observation of the processes and errors elicited during test performance, beyond the mere reliance on a single total achievement test score, afforded additional meaningful information to characterize the neuropsychological profile of the respondent. Further studies with clinical populations are needed in order to test the capacity of the MoCA-PA to distinguish between clinical subtypes of MCI that may be used to support a diagnostic process.

In order to aid the process of identification of the primary underlying neurocognitive mechanism responsible for defective test performance, without significantly affecting the original standardized administration procedures, the MoCAPA adds complementary or satellite test conditions in some of its subtests, includes "new" qualitative indices to capture the cognitive processes involved in each cognitive task and incorporates "new" qualitative classifications of error subtypes. In developing the MoCA-PA, we borrowed from the methods advanced by different authors who had previously helped to elaborate a neuropsychological conceptualization of the underlying cognitive constructs involved in the original cognitive tasks used in the standard MoCA, and had drawn different methods of qualitative error analysis and scoring.

The modification of "classic" cognitive instruments using the theoretical and methodological framework of the BPA (Kaplan, 1988) is not new and in fact many instruments have been adapted using this methodology in the past, and these modifications have proven of clinical and diagnostic utility. A prime example includes the Wechsler Adult Intelligence Scale-Revised as a Neuropsychological Instrument (WAIS-R-NI; Kaplan, Fein, Kramer, \& Delis, 1991) and the Delis-Kaplan Executive Function System
(D-KEFS; Delis et al., 2001), which, for the most part, represents a compilation of nine already existing classic tests of executive function.

Specific to the cognitive evaluation of older adults with the view of differentiating the effects of normal aging on cognition from the effects of stroke and different neurodegenerative conditions, the Kaplan-Baycrest Neurocognitive Assessment was purposely developed informed by BPA methodology (Leach, Kaplan, Rewilak, \& Proulx, 2000). Other cognitive tests have been developed informed by the BPA including, among others, the Boston Diagnostic Aphasia Examination (BDAE) (Goodglass \& Kaplan, 1983), the California Verbal Learning Test (Delis, Kramer, Kaplan, \& Ober, 1987, 2000), and the subsequently developed Philadelphia (repeatable) Verbal Learning Test (Price et al., 2011), developed for the assessment of memory in older adults. More recently, Filanosky and Kaplan (2006) adapted the Repeatable Battery for the Assessment of Neuropsychological Status (RBANS) (Randolph, Tierney, Mohr, \& Chase, 1998) using the BPA by developing an organized and systematic method for evaluating examinee's errors while also providing specific suggestions for testing limits.

In fact, in reference to the MoCA, Price et al. (2011) already demonstrated how supplementing the standard MoCA version of the command clock condition with a copy condition improved the clinical classification utility of this measure. Specifically, the authors found that whereas the clock drawn to command failed to differentiate individuals with dementia (i.e., AD, Vascular Dementia, and PD with Dementia) from controls and between dementia subtypes, specific cognitive patterns and diagnostic differences emerged when the command and copy performances were compared. However, to the best of our knowledge, the MoCA-PA represents the first adaptation of a brief cognitive screening instrument using the principles and methods of the PBA.

We believe that the MoCA-PA adds to the standard version in important ways that may prove to be useful in both clinical and research practice. Without adding any significant burden on the respondent, the qualitative version of the MoCA facilitates the concurrent assessment of multiple cognitive processes within each task by allowing the detailed tracking of qualitative aspects of performance, including types of responses and errors. This relative "burden" is placed on the examiner, who must carefully observe the strategies employed by respondents and systematically classify the qualitatively features of their test performance. The MoCA was designed to detect MCI but not to differentiate subtypes (e.g., MCI due to underlying AD versus subcortical small vessel ischemic changes). However, we believe that by incorporating the qualitative process approach methodology, the MoCA-PA may improve upon the standard version in terms of its clinical utility in differentiating the cognitive patterns of different MCI subtypes by helping to detect more subtle changes in cognitive test performance that would otherwise be obscured by the traditional quantitative 
scoring practices, which amalgamates the relative contribution of distinct cognitive processes into a single index score.

Nevertheless, while the literature attests to the clinical value of the application of the methodology derived from the BPA in the early detection and differential diagnosis of MCI subtypes (Ashendorf et al., 2013) it is fair to say that, like other previously modified versions of standard cognitive tests (e.g., D-KEFS Trail Making), further research is required to quantify the qualitative observations derived from the process approach methodology of the MoCA-PA, subjecting them to rigorous psychometric analysis (Crawford, Sutherland, \& Garthwaite, 2008; Erickson, 1995).

In relation to this, limitations of the current study are clear. As the MoCA-PA is still in development, this pilot study was conducted with healthy older adults in order to help us unveil those processes and strategies that might require to be examined in more detail before attempting to use the MoCA-PA with clinical samples. Thus, although the preliminary results are promising, the current study does not focus on the study of a clinical sample that could exemplify the neurocognitive mechanisms captured by MoCAPA. This work with clinical samples is ongoing and is expected to provide results in the near future. Moreover, and because the modifications proposed for the MoCA-PA are still under study, larger studies with both healthy and clinical samples are required before determining an appropriate scoring system (and interpretative guide) for the proposed additional indices. As it stands right now, the MoCA-PA provides the flexibility to use the standard 30point raw score format that professionals are familiar with, while at the same time allowing capture of additional information about the presence and nature of different processes and error subtypes. For example, frequency of different error types could obviously be registered quantitatively but the relevance of these errors needs, we believe, further study beyond mere quantification or simple scoring as present or absent. Additionally, in order to improve the detection of both false positives and false negatives in the screening stage, it is of great interest to our research group to identify those cases with a raw MoCA score below or above the standard cut-point (whatever those cut-points are determined to be), but whose qualitative performance may point in the opposite direction (e.g., score above the cut-point with indications of decline of clinical relevance). With all these procedures solved, it would be relatively straightforward to extrapolate the results obtained with the full MoCA-PA to create PBA versions for the MoCA-Blind (Wittich, Phillips, Nasreddine, \& Chertkow, 2010), MoCABasic (Julayanont et al., 2015) and s-MOCA (Roalf et al., 2016).

Furthermore, while the MoCA-PA may prove to be helpful in unraveling the cognitive profile of different clinical populations, it should not be used to replace detailed neuropsychological evaluation. However, in our attempt to systematically incorporate the process approach methodology to a widely used and proven clinically useful brief cognitive screening test, we hope that the MoCA-PA will bring this paradigm of neuropsychological test interpretation to a broader set of first-line clinicians. Ongoing and future research will aim to collect normative data for different clinical populations using the newly developed indices in order to determine the validity and clinical utility of the relatively novel qualitative process-based methods used in the MoCA-PA.

\section{Funding}

This work was supported by the European Commission, under the program MSCA-IF (Marie Sklodowska Curie Actions-Individual Fellowship), Grant Number 654895-E-SPACE-H2020-MSCA-IF-2014.

\section{ORCID}

Unai Diaz-Orueta (D) http://orcid.org/0000-0002-0349-8890

Ana Belen Navarro-Prados (ID http://orcid.org/0000-0003-1931-4619

\section{References}

Ahmed, S., Brennan, L., Eppig, J., Price, C. C., Lamar, M., DelanoWood, L., ... Libon, D. J. (2016). Visuoconstructional impairment in subtypes of mild cognitive impairment. Applied Neuropsychology: Adult, 23(1), 43-52. doi:10.1080/23279095.2014.1003067

Appels, B. A., \& Scherder, E. (2010). The diagnostic accuracy of dementia-screening instruments with an administration time of 10 to 45 minutes for use in secondary care: A systematic review. American Journal of Alzheimer's Disease \& Other Dementiasr, 25(4), 301-316. doi:10.1177/1533317510367485

Ashendorf, L. (2013). The trail making test. In L. Ashendorf, R. Swenson, \& D. Libon (Eds.), The Boston process approach to neuropsychological assessment: A practitioner's guide (pp. 111-121). New York, NY: Oxford University Press.

Ashendorf, L., Swenson, R., \& Libon, D. (2013). The Boston process approach to neuropsychological assessment: A practitioner's guide. New York, NY: Oxford University Press.

Benton, A. L., \& Hamsher, K. (1976). Multilingual aphasia examination (2nd ed.). Iowa City, IA: AJA Associates.

Biundo, R., Weis, L., Bostantjopoulou, S., Stefanova, E., FalupPecurariu, C., Kramberger, M. G., ... Aarsland, D. (2016). MMSE and MoCA in Parkinson's disease and dementia with Lewy bodies: A multicenter 1-year follow-up study. Journal of Neural Transmission, 123(4), 431-438. doi:10.1007/s00702-016-1517-6

Blanco-Campal, A., Coen, R., Diaz-Orueta, U., Irving, K., \& Burke, T. (2016). The Montreal Cognitive Assessment: A Qualitative Process Approach Version (MoCA-QPA). Poster presented at the 2016 midyear meeting of the International Neuropsychological Society, London, United Kingdom, 6th-8th July. Journal of the International Neuropsychological Society, 22 (Supp 2), 72.

Cacho, J., García-García, R., Arcaya, J., Vicente, J. L., \& Lantada, N. (1999). A proposal for application and scoring of the Clock Drawing Test in Alzheimer's disease [Article in Spanish]. Revista de Neurologia, 28(7), 648-655.

Cecato, J. F., Martinelli, J. E., Izbicki, R., Yassuda, M. S., \& Aprahamian, I. (2016). A subtest analysis of the Montreal Cognitive Assessment (MoCA): Which subtests can best discriminate between healthy controls, mild cognitive impairment and Alzheimer's disease? International Psychogeriatrics, 28(5), 825-832. doi:10.1017/ S1041610215001982

Coen, R. F., Cahill, R., \& Lawlor, B. A. (2011). Things to watch out for when using the Montreal Cognitive Assessment (MoCA). International Journal of Geriatric Psychiatry, 26(1), 107-108. doi:10. 1002/gps.2471

Coen, R. F., Robertson, D. A., Kenny, R. A., \& King-Kallimanis, B. L. (2016). Strengths and limitations of the MoCA for assessing 
cognitive functioning. Journal of Geriatric Psychiatry and Neurology, 29(1), 18-24. doi:10.1177/0891988715598236

Cordell, C. B., Borson, S., Boustani, M., Chodosh, J., Reuben, D., Verghese, J., ... Fried, L. B. (2013). Alzheimer's association recommendations for operationalizing the detection of cognitive impairment during the Medicare Annual Wellness Visit in a primary care setting. Alzheimer's \& Dementia, 9(2), 141-150. doi:10.1016/j.jalz. 2012.09.011

Cosentino, S., Jefferson, A., Chute, D. L., Kaplan, E., \& Libon, D. J. (2004). Clock drawing errors in dementia: Neuropsychological and neuroanatomical considerations. Cognitive and Behavioral Neurology, 17(2), 74-84. doi:10.1097/01.wnn.0000119564.08162.46

Costa, A. S., Fimm, B., Friesen, P., Soundjock, H., Rottschy, C., Gross, T., ... Reetz, K. (2012). Alternate-form reliability of the Montreal Cognitive Assessment screening test in a clinical setting. Dementia and Geriatric Cognitive Disorders, 33(6), 379-384. doi:10.1159/ 000340006

Crawford, J. R., Sutherland, D. M., \& Garthwaite, P. H. (2008). On the reliability and standard errors of measurement of contrast measures from the D-KEFS. Journal of the International Neuropsychological Society, 14(6), 1069-1073. doi:10.1017/S1355617708081228

Cullen, B., O’Neill, B., Evans, J. J., Coen, R. F., \& Lawlor, B. A. (2007) A review of screening tests for cognitive impairment. Journal of Neurology, Neurosurgery, and Psychiatry, 78(8), 790-799. doi:10. 1136/jnnp.2006.095414

Dagenais, E., Rouleau, I., Demers, M., Jobin, C., Roger, E., Chamelian, L., \& Duquette, P. (2013). Value of the MoCA test as a screening instrument in multiple sclerosis. Canadian Journal of Neurological Sciences/Journal Canadien Des Sciences Neurologiques, 40(3), 410-415. doi:10.1017/S0317167100014384

Dalrymple-Alford, J. C., MacAskill, M. R., Nakas, C. T., Livingston, L., Graham, C., Crucian, G. P., ... Anderson, T. J. (2010). The MoCA well-suited screen for cognitive impairment in Parkinson disease. Neurology, 75(19), 1717-1725. doi:10.1212/WNL.0b013e3181fc29c9

Davis, D. H. J., Creavin, S. T., Yip, J. L. Y., Noel-Storr, A. H., Brayne, C., \& Cullum, S. (2015). Montreal Cognitive Assessment for the diagnosis of Alzheimer's disease and other dementias. Cochrane Database of Systematic Reviews 2015, 10, CD010775. doi:10.1002/ 14651858.CD010775.pub2

Delis, D. C., Kaplan, E., \& Kramer, J. H. (2001). Delis-Kaplan executive function system (D-KEFS). San Antonio, TX: The Psychological Corporation.

Delis, D. C., Kramer, J. H., Kaplan, E., \& Ober, B. A. (1987). California verbal learning test: Adult version (Manual). San Antonio, TX Psychological Corporation

Delis, D. C., Kramer, J. H., Kaplan, E., \& Ober, B. A. (2000). California verbal learning test (2nd ed., Adult version, Manual). San Antonio, TX: Psychological Corporation.

Dong, Y., Sharma, V. K., Chan, B. P.-L., Venketasubramanian, N., Teoh, H. L., Seet, R. C. S., ... Chen, C. (2010). The Montreal Cognitive Assessment (MoCA) is superior to the Mini-Mental State Examination (MMSE) for the detection of vascular cognitive impairment after acute stroke. Journal of the Neurological Sciences, 299(1-2), 15-18. doi:10.1016/j.jns.2010.08.051

Erickson, R. C. (1995). A review and critique of the process approach in neuropsychological assessment. Neuropsychology Review, 5(4), 223-243. doi:10.1007/BF02214647

Evans, I., Coen, R., Burke, T., \& Lawlor, B. A. (2005). Clock drawing and executive function in Alzheimer's disease. Conference Paper. Irish Journal of Medical Science, 174(3, Supp.2), 80-80.

Fengler, S., Kessler, J., Timmermann, L., Zapf, A., Elben, S., Wojtecki, L., $\ldots$ Kalbe, E. (2016). Screening for cognitive impairment in Parkinson's Disease: Improving the diagnostic utility of the MoCA through subtest weighting. Plos One, 11(7), e0159318. doi:10.1371/ journal.pone. 0159318

Filanosky, C., \& Kaplan, E. (2006). Applying the Boston process approach to the RBANS. Poster presented at the 4th Annual
Conference of the American Academy of Clinical Neuropsychology. Philadelphia, PA.

Folstein, M.F., Folstein, S.E., \& McHugh, P.R. (1975). "Mini-mental state". A practical method for grading the cognitive state of patients for the clinician. Journal of Psychiatric Research, 12(3), 189-198.

Freedman, M., Leach, L., Kaplan, E., Winocur, G., Shulman, K., \& Delis, D. (1994). Clock drawing: A neuropsychological approach. New York, NY: Oxford University Press.

Freitas, S., Santana, S., \& Simoes, M. R. (2010). The sensitivity of the MoCA and MMSE to cognitive decline: A longitudinal study. Alzheimer's \& Dementia, 6(4), S353-S354. doi:10.1016/j.jalz.2010.05. 1184

Freitas, S., Simoes, M. R., Alves, L., Duro, D., \& Santana, I. (2012). Montreal Cognitive Assessment (MoCA): Validation study for frontotemporal dementia. Journal of Geriatric Psychiatry and Neurology, 25(3), 146-154. doi:10.1177/0891988712455235

Freitas, S., Simoes, M. R., Alves, L., Vicente, M., \& Santana, I. (2012). Montreal Cognitive Assessment (MoCA): Validation study for vascular dementia. Journal of the International Neuropsychological Society, 18(6), 1031-1040. doi:10.1017/S135561771200077X

Freitas, S., Simoes, M. R., Maroco, J., Alves, L., \& Santana, I. (2012). Construct validity of the Montreal Cognitive Assessment. Journal of the International Neuropsychological Society, 18(2), 242-250. doi:10. $1017 /$ S1355617711001573

Gagnon, G., Hansen, K. T., Woolmore-Goodwin, S., Gutmanis, I., Wells, J., Borrie, M., \& Fogarty, J. (2013). Correcting the MoCA for education: Effect on sensitivity. Canadian Journal of Neurological Sciences / Journal Canadien Des Sciences Neurologiques, 40(5), 678-683. doi:10.1017/S0317167100014918

Geslani, D. M., Tierney, M. C., Herrman, N., \& Szalai, J. P. (2005). Mild cognitive impairment: An operation definition and its conversion rate to Alzheimer's disease. Dementia and Geriatric Cognitive Disorders, 19(5-6), 383-389. doi:10.1159/000084709

Goodglass, H., \& Kaplan, E. (1983). The assessment of aphasia and related disorders (Vol. 2). Philadelphia, PA: Lea \& Felbiger.

Hanagasi, H. A., Gurvit, H., Unsalan, P., Horozoglu, H., Tuncer, N., Feyzioglu, A., ... Emre, M. (2011). The effects of rasagiline on cognitive deficits in Parkinson's disease patients without dementia: A randomized, double-blind, placebo-controlled, multicenter study. Movement Disorders, 26(10), 1851-1858. doi:10.1002/mds.23738

Henry, J. D., Crawford, J. R., \& Phillips, L. H. (2004). Verbal fluency performance in dementia of the Alzheimer's type: A meta-analysis. Neuropsychologia, 42(9), 1212-1222.

Hodges, J. R., Salmon, D. P., \& Butters, N. (1991). The nature of the naming deficit in Alzheimer's and Huntington's disease. Brain, 114(4), 1547-1558.

Hoops, S., Nazem, S., Siderowf, A. D., Duda, J. E., Xie, S. X., Stern, M. B., \& Weintraub, D. (2009). Validity of the MoCA and MMSE in the detection of MCI and dementia in Parkinson disease. Neurology, 73(21), 1738-1745. doi:10.1212/WNL.0b013e3181c34b47

Horton, D. K., Hynan, L. S., Lacritz, L. H., Rossetti, H. C., Weiner, M. F., \& Cullum, C. M. (2015). An abbreviated Montreal Cognitive Assessment (MoCA) for dementia screening. The Clinical Neuropsychologist, 29(4), 413-425. / doi:10.1080/13854046.2015. 1043349

Julayanont, P., \& Nasreddine, Z. S. (2017). Montreal Cognitive Assessment (MoCA): Concept and clinical review. In A. J. Larner (Ed.), Cognitive screening instruments (pp. 139-195). Cham, Switzerland: Springer.

Julayanont, P., Tangwongchai, S., Hemrungrojn, S., Tunvirachaisakul, C., Phanthumchinda, K., Hongsawat, J., ... Nasreddine, Z. S. (2015). The Montreal Cognitive Assessment-Basic (MoCA-B): A new mild cognitive impairment screening test for illiterate and low educated elderly. Journal of the American Geriatrics Society, 63(12), 2550-2554. doi:10.1111/jgs.13820

Kaplan, E. (1988). A process approach to neuropsychological assessment. In T. Boll \& B. K. Bryant (Eds.). Clinical neuropsychology and brain function: Research, measurement, and practice. Washington, 
DC: American Psychological Association.

Kaplan, E. (1990). The process approach to neuropsychological assessment of psychiatric patients. Journal of Neuropsychiatry and the Clinical Neurosciences, 2, 72-87.

Kaplan, E., Fein, D., Kramer, J., \& Delis, D. (1991). WAIS-R as a neuropsychological instrument (WAIS-R-NI). San Antonio, TX: The Psychological Corporation.

Kaufman, D. A. S., Boxer, O., \& Bilder, R. M. (2013). Evidence-based science and practice in neuropsychology: A review. In S. Koffler, J. Morgan, I. S. Baron, \& M. F. Greiffenstein (Eds.). Neuropsychology: Science and practice, I. New York, NY: Oxford University Press.

Kenny, R. A., Coen, R. F., Frewen, J., Donoghue, O. A., Cronin, H., \& Savva, G. M. (2013). Normative values of cognitive and physical function in older adults: Findings from the Irish longitudinal study on ageing. Journal of the American Geriatrics Society, 61(Suppl 2), S279-S290. doi:10.1111/jgs.12195

Lamar, M., Rhodes, E., Wambach, D. M., Swenson, R., \& Libon, D. J. (2013). Digit Span as a neuropsychological instrument of error processing. In L. Ashendorf, R. Swenson, \& D. Libon (Eds.), The Boston process approach to neuropsychological assessment: A practitioner's guide (pp. 65-76). New York, NY: Oxford University Press.

Larner, A. J. (2012). Screening utility of the Montreal Cognitive Assessment (MoCA): In place of -or as well as -the MMSE? International Psychogeriatrics, 24(3), 391-396. doi:10.1017/ S1041610211001839

Leach, L., Kaplan, E., Rewilak, D., \& Proulx, G. B. (2000). Kaplan Baycrest Neurocognitive Assessment (KBNA). San Antonio, TX: Pearson Education.

Libon, D. J., Malamut, B. L., Swenson, R., Sands, L. P., \& Cloud, B. S. (1996). Further analysis of clock drawings among demented and non-demented subjects. Archives of Clinical Neuropsychology, 11(3), 193-211. doi:10.1093/arclin/11.3.193

Luis, C. A., Keegan, A. P., \& Mullan, M. (2009). Cross validation of the Montreal Cognitive Assessment in community dwelling older adults residing in the Southeastern US. International Journal of Geriatric Psychiatry, 24(2), 197-201. doi:10.1002/gps.2101

Luria, A. R. (1980). Language and cognition. New York, NY: John Wiley and Sons.

Mai, L. M., Oczkowski, W., Mackenzie, G., Shuster, A., Wasielesky, L., Franchetto, A., ... Sahlas, D. J. (2013). Screening for cognitive impairment in a stroke prevention clinic using the MoCA. Canadian Journal of Neurological Sciences/Journal Canadien Des Sciences Neurologiques, 40(2), 192-197. Retrieved from http://www. ncbi.nlm.nih.gov/pubmed/23419567 doi:10.1017/S031716710001372X

Malek-Ahmadi, M., Powell, J. J., Belden, C. M., O'Connor, K., Evans, L., Coon, D. W., \& Nieri, W. (2015). Age- and education-adjusted normative data for the Montreal Cognitive Assessment (MoCA) in older adults age 70-99. Neuropsychology, Development, and Cognition. Section B, Aging, Neuropsychology and Cognition, 5585, $1-7$.

Milberg, W. P., Hebben, N. A., \& Kaplan, E. (1986). The Boston process approach to neuropsychological assessment. In G. Adams (Ed.) Neuropsychological assessment of neuropsychiatric disorders (pp. 42-65). New York, NY: Oxford University Press.

Milne, A., Culverwell, A., Guss, R., Tuppen, J., \& Whelton, R. (2008) Screening for dementia in primary care: A review of the use, efficacy and quality of measures. International Psychogeriatrics, 20(05), 911-926. doi:10.1017/S1041610208007394

Nasreddine, Z. S., Phillips, N. A., Bã CDirian, VÃ C R., Charbonneau, S., Whitehead, V., Collin, I., ... Chertkow, H. (2005). The Montreal Cognitive Assessment, MoCA: A brief screening. Journal of the American Geriatrics Society, 53(4), 695-699. doi:10.1111/j.1532-5415. 2005.53221.x

Ojeda, N., Del Pino, R., Ibarretxe-Bilbao, N., Schretlen, D. J., \& Pena, J. (2016). Montreal Cognitive Assessment Test: Normalization and standardization for Spanish population [Article in Spanish]. Revista de Neurologia, 63(11), 488-496.

Paul, R., Lane, E. M., Tate, D. F., Heaps, J., Romo, D. M., Akbudak, E., Niehoff, J., \& Conturo, T. E. (2011). Neuroimaging signatures and cognitive correlates of the Montreal Cognitive Assessment screen in a nonclinical elderly sample. Archives of Clinical Neuropsychology, 26(5), 454-460. doi:10.1093/arclin/acr017

Pendlebury, S. T., Mariz, J., Bull, L., Mehta, Z., \& Rothwell, P. M. (2012). MoCA, ACE-R, and MMSE versus the National Institute of Neurological Disorders and Stroke-Canadian Stroke Network vascular cognitive impairment harmonization standards neuropsychological battery after TIA and stroke. Stroke, 43(2), 464-469. doi:10. 1161/STROKEAHA.111.633586

Petersen, R. C., Doody, R., Kurz, A., Mohs, R. C., Morris, J. C., Rabins, P. V., ... Winblad, B. (2001). Current concepts in mild cognitive impairment. Archives of Neurology, 58(12), 1985-1992. doi:10.1001/ archneur.58.12.1985

Poreh, A. (2000). The quantified process approach: An emerging methodology to neuropsychological assessment. The Clinical Neuropsychologist, 14(2), 212-222.

Poreh, A. (2006). The quantified process approach to neuropsychological assessment. New York, NY: Taylor \& Francis.

Price, C. C., Cunningham, H., Coronado, N., Freedland, A., Cosentino, S., Penney, D. L., ... Libon, D. J. (2011). Clock drawing in the Montreal Cognitive Assessment: Recommendations for dementia assessment. Dementia and Geriatric Cognitive Disorders, 31(3), 179-187. doi:10.1159/000324639

Randolph, C., Tierney, M. C., Mohr, E., \& Chase, T. N. (1998). The Repeatable Battery for the Assessment of Neuropsychological Status (RBANS): Preliminary clinical validity. Journal of Clinical and Experimental Neuropsychology, 20(3), 310-319. doi:10.1076/jcen.20.3. 310.823

Reitan, R. M., \& Wolfson, D. (1992). Neuropsychological evaluation of older children. Tucson, AZ: Neuropsychology Press.

Roalf, D. R., Moore, T. M., Wolk, D. A., Arnold, S. E., MechanicHamilton, D., Rick, J., ... Moberg, P. J. (2016). Defining and validating a short form Montreal Cognitive Assessment (s-MoCA) for use in neurodegenerative disease. Journal of Neurology, Neurosurgery \& Psychiatry, 87(12), 1303-1310. doi:10.1136/jnnp-2015-312723

Rossetti, H. C., Lacritz, L. H., Cullum, C. M., \& Weiner, M. F. (2011). Normative data for the Montreal Cognitive Assessment (MoCA) in a population-based sample. Neurology, 77(13), 1272-1275. doi:10. 1212/WNL.0b013e318230208a

Rouleau, I., Salmon, D. P., \& Butters, N. (1996). Longitudinal analysis of clock drawing in Alzheimer's disease patients. Brain and Cognition, 31(1), 17-34. doi:10.1006/brcg.1996.0022

Rouleau, I., Salmon, D. P., Butters, N., Kennedy, C., \& McGuire, K. (1992). Quantitative and qualitative analyses of clock drawings in Alzheimer's and Huntington's disease. Brain and Cognition, 18(1), 70-87. doi:10.1016/0278-2626(92)90112-Y

Ryan, J. P., McGowan, J., McCaffrey, N., Ryan, G. T., Zandi, T., \& Brannigan, G. G. (1995). Graphomotor perseveration and wandering in Alzheimer's disease. Journal of Geriatric Psychiatry and Neurology, 8, 209-212. doi:10.1177/089198879500800402

Smith, T., Gildeh, N., \& Holmes, C. (2007). The Montreal Cognitive Assessment: validity and utility in a memory clinic setting. The Canadian Journal of Psychiatry, 52(5), 329-332. doi:10.1177/ 070674370705200508

Troyer, A. K. (2000). Normative data for clustering and switching on verbal fluency task. Journal of Clinical and Experimental Neuropsychology, 22(3), 370-378. doi:10.1076/13803395(200006)22:3;1-V;FT370

Troyer, A. K., Moscovitch, M., \& Winocur, G. (1997). Clustering and switching as two components of verbal fluency: Evidence from younger and older healthy adults. Neuropsychology, 11(1), 138-146. doi:10.1037/0894-4105.11.1.138

Vaughan, R. M., Coen, R. F., Kenny, R. A., \& Lawlor, B. A. (2016). Preservation of the semantic verbal fluency advantage in a large population-based sample: Normative data from the TILDA study. Journal of the International Neuropsychological Society, 22(5), 570-576. doi:10.1017/S1355617716000291

Waldron-Perrine, B., \& Axelrod, B. N. (2012). Determining an appropriate cutting score for indication of impairment on the Montreal Cognitive Assessment. International Journal of Geriatric Psychiatry, 27(11), 1189-1194. doi:10.1002/gps.3768 
Wittich, W., Phillips, N., Nasreddine, Z. S., \& Chertkow, H. (2010). Sensitivity and specificity of the Montreal Cognitive Assessment modified for individuals who are visually impaired. Journal of Visual Impairment \& Blindness, 104(6), 360-368. doi:10.1177/ $0145482 X 1010400606$
Zadikoff, C., Fox, S. H., Tang-Wai, D. F., Thomsen, T., de Bie, R. M., Wadia, P., ... Marras, C. (2008). A comparison of the mini mental state exam to the Montreal cognitive assessment in identifying cognitive deficits in Parkinson's disease. Movement Disorders, 23(2), 297-299. doi:10.1002/mds.21837 